Géographie physique et Quaternaire

\title{
L'environnement tardiglaciaire du Yukon septentrional, Canada
}

\section{Late-Glacial Environment in Northern Yukon Окружающая среда северного юкона в позднеледниковый период}

\author{
J. C. Ritchie, J. Cinq-Mars et L. C. Cwynar
}

Volume 36, numéro 1-2, 1982

URI : https://id.erudit.org/iderudit/032480ar

DOI : https://doi.org/10.7202/032480ar

Aller au sommaire du numéro

Éditeur(s)

Les Presses de l'Université de Montréal

ISSN

0705-7199 (imprimé)

1492-143X (numérique)

Découvrir la revue

Citer cet article

Ritchie, J. C., Cinq-Mars, J. \& Cwynar, L. C. (1982). L’environnement tardiglaciaire du Yukon septentrional, Canada. Géographie physique et Quaternaire, 36(1-2), 241-250. https://doi.org/10.7202/032480ar
Résumé de l'article

Le site des grottes du Poisson-Bleu, localisé sur une assise à calcaires dévoniens au sein de la forêt boréale du Yukon septentrional, a fourni, en plus d'un abondant matériel paléontologique et de quelques données archéologiques, des sédiments pollinifères. Le diagramme pollinique d'un dépôt consistant en un loess tardiglaciaire recouvert d'un humus à cailloutis d'âge holocène présente un assemblage inférieur dominé par des herbes de toundra et un assemblage supérieur dominé par l'épinette et l'aulne. Grâce à ces données ainsi qu'à celles tirées de sites avoisinants, il est possible d'en arriver à une reconstitution de la végétation dont les phases importantes sont les suivantes: une période tardiglaciaire, de 16000 à $12000 \mathrm{BP}$, caractérisée par une toundra herbacée, éparse sur les hauts plateaux et par des marécages à laiches et à graminées dans les basses terres; ultérieurement, au cours de l'Holocène, le phénomène d'entourbement du sol et le développement de bruyères et de pessières, conséquences d'un climat plus chaud. 


\section{L'ENVIRONNEMENT TARDIGLACIAIRE DU YUKON SEPTENTRIONAL, CANADA}

J.C. RITCHIE, J. CINQ-MARS et L.C. CWYNAR, respectivement, Scarborough College, University of Toronto, Toronto, Ontario M1C 1A4, Commission archéologique du Canada. Musée national de l'Homme, Ottawa, Ontario K1A 0M8 et Quaternary Research Center, University of Washington, AK-60, Seattle, Washington 98195, U.S.A.

RÉSUMÉ Le site des grottes du Poisson-BLeu, localisé sur une assise à calcaires dévoniens au sein de la forêt boréale du Yukon septentrional, a fourni, en plus d'un abondant matériel paléontologique et de quelques données archéologiques, des sédiments pollinifères. Le diagramme pollinique d'un dépôt consistant en un lœss tardiglaciaire recouvert d'un humus à cailloutis d'âge holocène présente un assemblage inférieur dominé par des herbes de toundra et un assemblage supérieur dominé par l'épinette et l'aulne. Grâce à ces données ainsi qu'à celles tirées de sites avoisinants, il est possible d'en arriver à une reconstitution de la végétation dont les phases importantes sont les suivantes: une période tardiglaciaire, de 16000 à 12000 $\mathrm{BP}$, caractérisée par une toundra herbacée, éparse sur les hauts plateaux et par des marécages à laiches et à graminées dans les basses terres; ultérieurement, au cours de l'Holocène, le phénomène d'entourbement du sol et le développement de bruyères et de pessières, conséquences d'un climat plus chaud.
ABSTRACT Late-glacial environment in northern Yukon. The Bluefish Caves site, located on a scarp of Devonian limestone in the northern boreal forest of the Yukon, has yielded pollen-bearing sediments in addition to abundant paleontological and some archaeological data. A pollen diagram based on samples of late-glacial lœss overlain by Holocene humus shows two main pollen assemblages, the lower dominated by herbs and dwarf shrubs and the upper by spruce and alder. Based on these results, but also drawing on recent findings from other sites in N. Yukon, we offer the following reconstruction of the vegetation history of the region. The vegetation of the late-glacial period, from 16000 to $12000 \mathrm{BP}$, was a sparse herb tundra on upland surfaces and a complex of sedgegrass marshes with willow on lowlands. There was a notable change in cover at the beginning of the Holocene when spruce forest spread to all upland surfaces except the highest ridges, and paludification in the lowlands resulted in the spread of bog and mire communities.
РЕЗЮМЕ Окр!жающая среда северного Юкона в позднеледниковый период. В районе расположения пещер Блуфиш, находящихся на крутом откосе известняка девонского периода в северном бореальном лесу Юкона, были обнаружены отложения, содержавшие пыльцу в дополнение к многочисленным палеонтологическим и археологическим данным. Диаграмма пыльцы, составленная на основе образцов, обнаруженных в позднеледниковом лессе, сверху которого находился перегной голоцена, показывает два главных скопления пыльцы: нижнее, в котором преобладают травы и карликовые кустарники и верхнее, в котором преобладают ель и ольха черная. Основываясь на этих результатах, а также используя недавние находки из других мест северного Юкона, мы предлагаем следующую историю растительности района. Растительность позднеледникового периода с 16000 до 12000 года до настоящего времени (до 1950 года нашей эры)* представляла собой тундру с редким кустарником на возвышенных местах и комплекс болот, поросших осокой и ивой в низинах. В начале голоцена прфизошла заметная перемена растительности, когда хвойный лес распространился на все возвышенности. кроме самых высоких хребтов, а заболачивание низменностей привело к распространению болотной растительности

*Примсчание шерево;чика 


\section{INTRODUCTION}

Malgré les efforts récents de plusieurs équipes de recherche, la préhistoire de la Béringie orientale demeure mal documentée (MORLAN, 1980). Semblablement, les essais de reconstitution de l'histoire géologique et environnementale de la région n'ont avancé que très lentement, d'où de nombreuses difficultés lorsqu'il s'est agi d'intégrer les données culturelles à l'évolution du milieu physique. Toutefois, la découverte de spécimens paléontologiques, botaniques et culturels dans les grottes du Poisson-Bleu (CINQ-MARS, 1979) rend maintenant possible une tentative de reconstitution de l'environnement tardiglaciaire du Yukon septentrional.

Depuis longtemps, on emploie de façon efficace l'analyse pollinique des sédiments sous grottes et abris pour la reconstitution de l'environnement du Pléistocène supérieur en Europe et ce, particulièrement dans le sud de la France (De LUMLEY et al., 1973; RENAULT-MISKOVSKY, 1978). On connaît bien, cependant, les limitations et les risques de cette méthode: irrégularités ou lacunes stratigraphiques des dépôts, existence de mélanges polliniques dus à des facteurs éoliens, hydrologiques et gravitationnels, ou encore reliées aux phénomènes de bioturbation et de géliturbation et, enfin, mauvaise conservation des grains de pollen. En conséquence, il est essentiel de compléter les données obtenues d'un gisement sous grotte ou abri par les résultats des travaux effectués sur des sites avoisinants dont les sédiments ne sont ni remaniés, ni contaminés. C'est la méthode que nous utilisons dans la présente étude, à savoir, l'interprétation du diagramme de la grotte du Poisson-Bleu II en relation avec les données issues de deux dépôts lacustres (le site Hanging L., à 200 km au nord-est des grottes du Poisson-Bleu et le site Lateral Pond à Doll Creek, à 200 km à l'est-sud-est) et d'une tourbière de la même région (le site Polybog, dans la plaine d'Old Crow, à 80 km à l'est-nord-est des grottes) (fig. 1).

Il est particulièrement approprié de comparer le site de Doll Creek avec celui du Poisson-Bleu parce que les deux ont en commun un paysage constitué de massifs calcaires non glaciés et un couvert arbustif et herbacé bien caractéristique des sols développés sur des carbonates. De même, ils sont tous deux situés aux limites de la forêt subarctique, dominée partout par l'épinette, sauf sur les crêtes et dans les basses terres humides.

Des descriptions détaillées des grottes du PoissonBleu (CINQ-MARS, 1979), des sites de Hanging L. et Doll Creek (CWYNAR, 1982; 1982; CWYNAR et RITCHIE, 1980 ; RITCHIE, 1982) ainsi que du site Polybog (OVENDEN, 1981) ont été présentées ailleurs. Nous résumerons donc ici quelques-unes des données relatives aux fouilles effectuées aux gisements du Poisson-Bleu, nous en exposerons la stratigraphie pollinique et, enfin, nous tenterons de reconstituer, avec l'apport des résul-

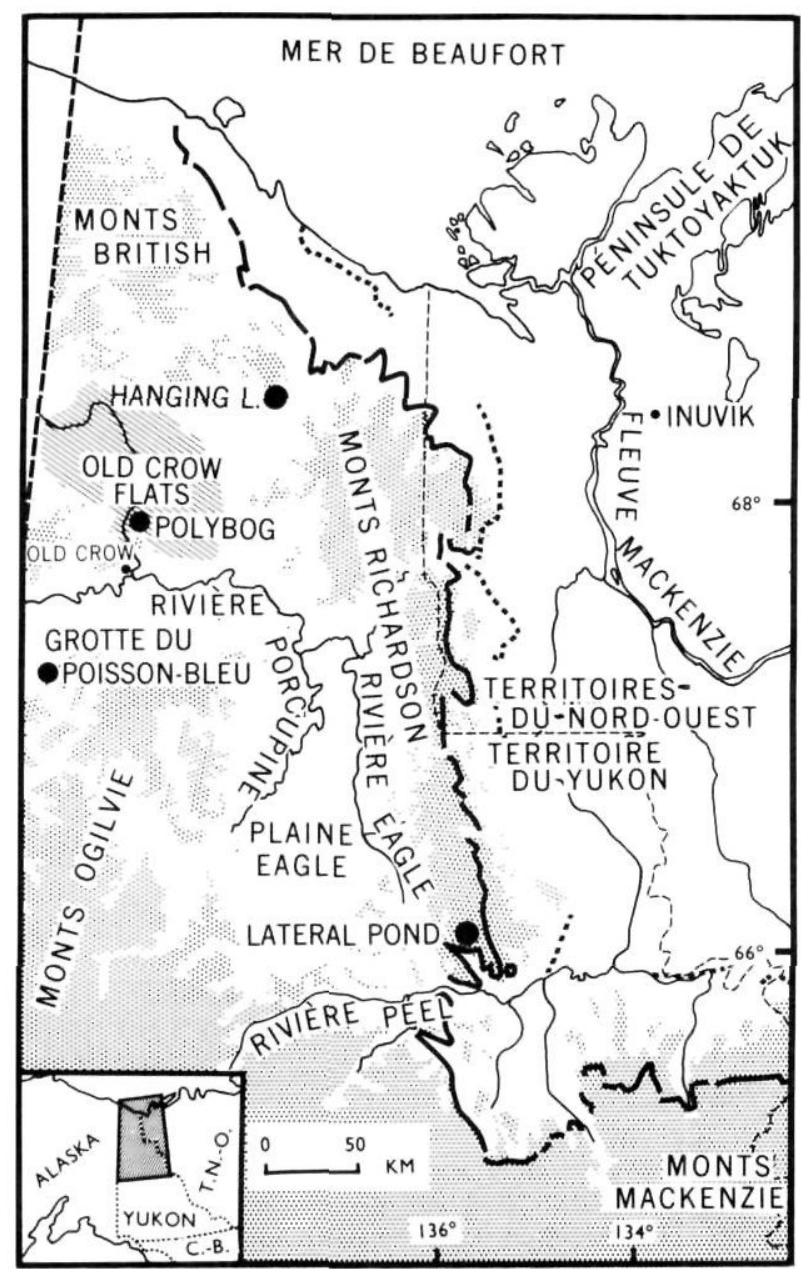

FIGURE 1. Carte de localisation des sites étudiés.

Location map showing the sites referred to in the text.

tats obtenus des autres sites, l'environnement de la période représentée par ces échantillons.

\section{DESCRIPTION DES GROTTES DU POISSON-BLEU}

Le site archéologique du Poisson-Bleu $\left(67^{\circ} 09^{\prime} \mathrm{N}\right.$ et $140^{\circ} 45^{\prime} \mathrm{O}$ ) est localisé sur une crête saillante dominant la rive droite du cours moyen de la rivière du Poisson-Bleu, un tributaire de la rivière Porcupine (fig. 2). Les grottes, de faibles dimensions, sont au nombre de trois. On les trouve à la base d'un escarpement calcaire discontinu où elles semblent s'être formées en partie par dissolution, mais surtout par gélifraction de poches de brèche - vestiges probables du paléoremplissage d'un ancien réseau karstique (fig. 3).

Les sondages et les fouilles effectués sous grottes et sur les replats en face des abris ont fourni, en plus des sédiments pollinifères, une abondance de restes paléontologiques, associés dans certains cas à des tra- 


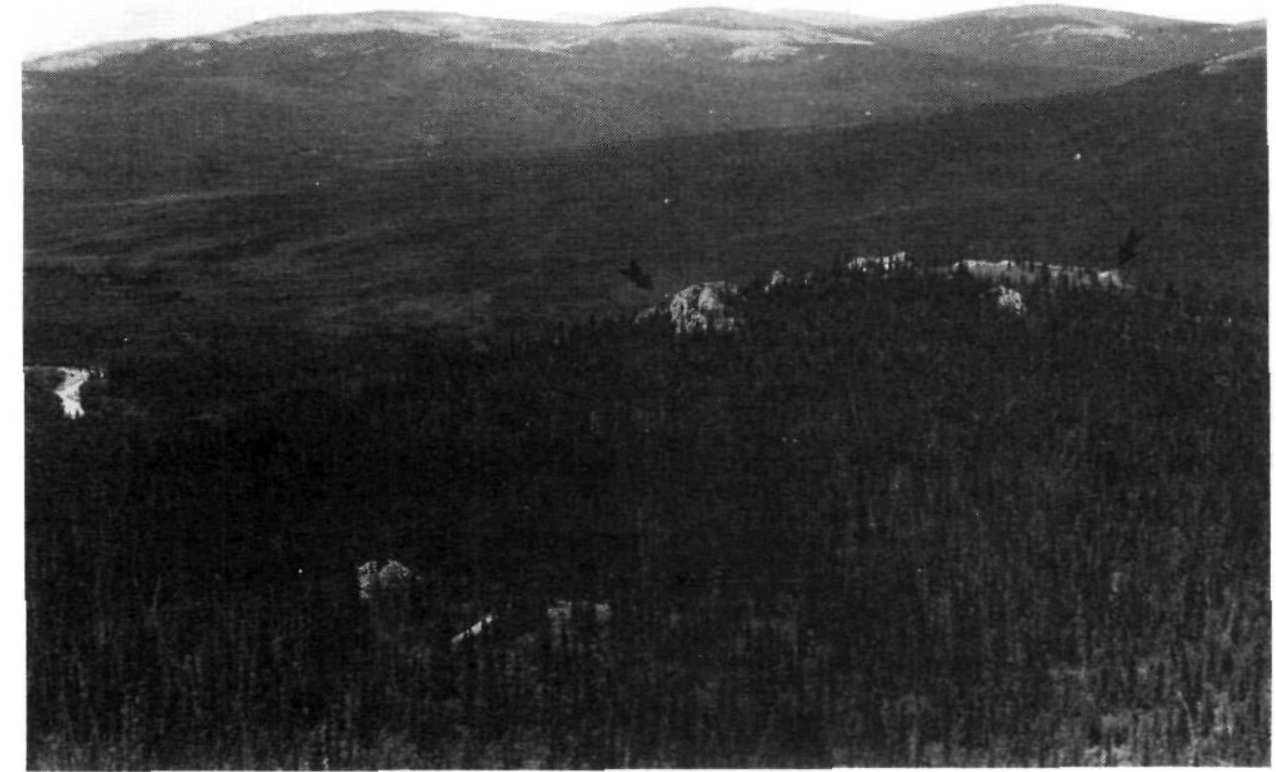

FIGURE 2. Vue aérienne de la crête et des affleurements calcaires où se trouvent les grottes du PoissonBleu. La rivière du même nom coule du sud au nord (de gauche à droite) et le sommet des collines que l'on voit en arrière plan correspond approximativement à la frontière de I'Alaska. La flèche de droite indique la position des grottes I et II et celle de gauche, la position de la grotte III. Le paysage végétal prédominant est la pessière, sauf sur les crêtes élevées où se trouve la toundra. Cette prédominance de la pessière est typique des régions non glaciées, à substrat calcaire, et diffère notablement des régions du Yukon septentrional et d'Alaska où les roches siliceuses portent des forêts mixtes, composées d'épinettes, de peupliers et de bouleaux.

An aerial view of the ridge and limestone outcrops on which the Bluefish Caves occur. The river of the same name flows from south to north (left to right) and the summits of the hills in the background coincide roughly with the Alaska border. The arrow on the right indicates the position of caves I and II, and the one on the left that of Cave III. The landscape is dominated by a spruce forest, with the exception of higher summit ridges where tundra prevails. This predominance of spruce is typical of unglaciated areas with calcareous bedrock, and contrasts

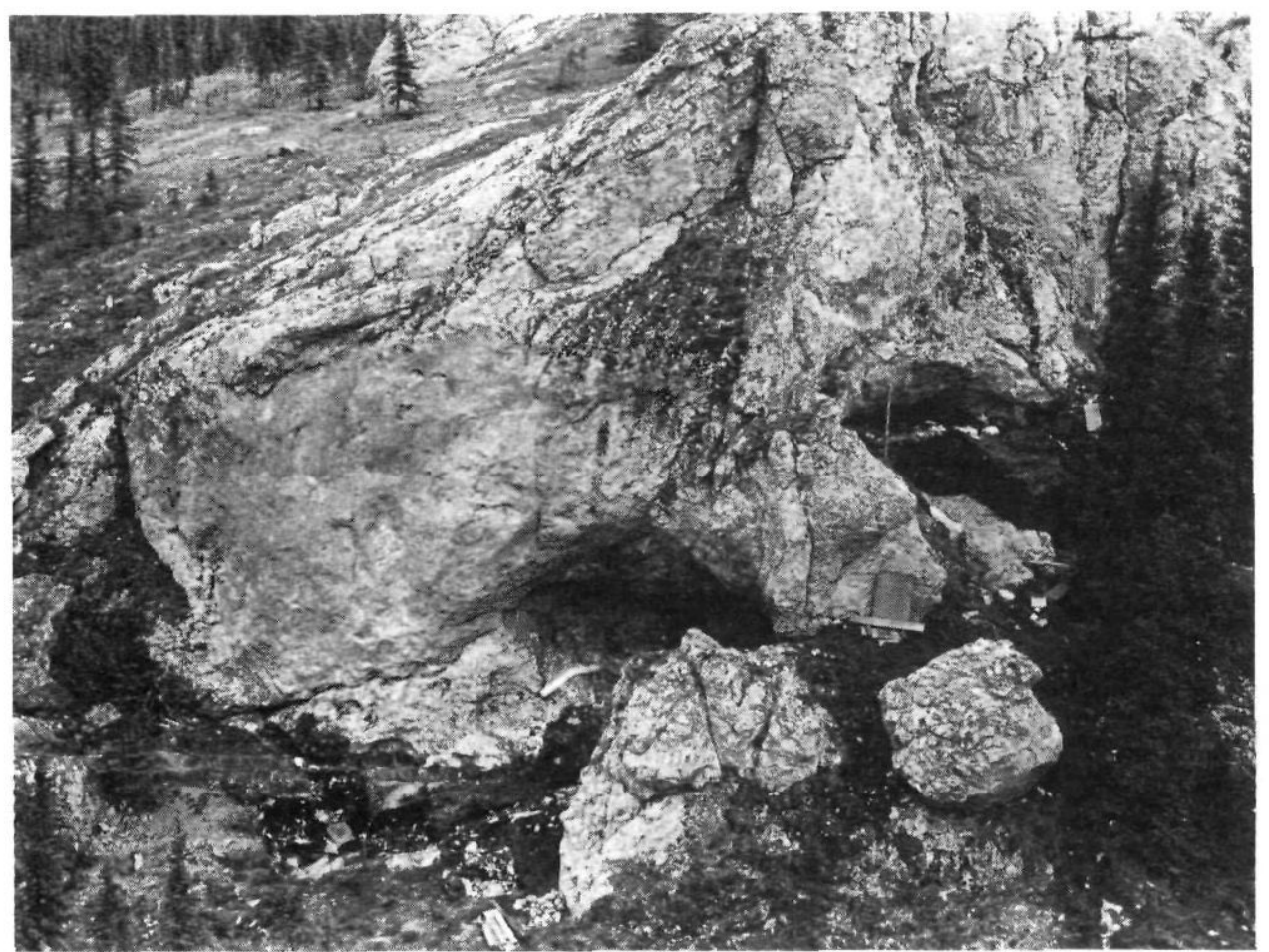
strongly with areas of the northern Yukon and Alaska where siliceous rock types support mixed forests of spruce, poplar and birch.

FIGURE 3. Vue aérienne des grottes I (à gauche) et II (à droite) ains que des zones de sondages et de fouilles effectués en 1979 et 1981.

An aerial view of Cave I (to the left) and Cave II (to the right) as well as some of the test pits and excavations made in 1979 and 1981. 
ces d'activités culturelles. En bref, la coupe stratigraphique se décrit comme suit: a) un substrat rocheux, plus ou moins gélifracté, b) un lœss à cailloutis, c) un humus à cailloutis et, enfin, d) le sol récent (fig. 4). Cette représentation schématique illustre bien les dépôts associés aux trois grottes. Les études sédimentologiques et pédologiques préliminaires (CINQ-MARS 1979, fig. 11) ainsi que celles qui sont en cours nous permettent d'entrevoir une séquence de dépôts composée d'un épisode éolien relativement homogène se terminant plutôt abruptement, suivi de façon parfois discordante selon les zones excavées par la mise en place de la couche d'humus. Le cailloutis incorporé au lœss aussi bien qu'à l'humus consiste en une accumulation, peut-être rythmique ou périodique, de roches gélifractées provenant des plafonds et des parois des grottes ainsi que des surplombs rocheux.

Les vestiges fauniques, qui ont été recueillis, se divisent en deux groupes correspondant aux deux plus importantes couches stratigraphiques. Le lœss à cailloutis a fourni une multitude de restes d'une faune vertébrée de type tardiglaciaire (HARINGTON, 1978), caractérisée entre autres par le cheval Equus cf. lambei), le mammouth (Mammuthus sp.) le mouflon (Ovis cf. dalli), le caribou (Rangifer tarandus), le bison (Bison sp.), le wapiti ou cerf élaphe (Cervus elaphus), le bœuf musqué (Ovibos) ainsi que par une grande variété de petits mammifères. On y trouve, de plus, des restes d'oiseaux divers et des fragments de poissons. Par ailleurs, la zone d'humus à cailloutis n'a fourni que des éléments représentatifs d'une faune de type moderne, c'est-à-dire appauvrie, caractérisée dans ce cas-ci par le caribou et par de nombreux micro-mammifères.

Quant au matériel culturel, on le retrouve, à une exception près, dans les sédiments éoliens. II s'agit avant tout de quelques éclats de chert, de petits galets, d'un burin d'angle sur la lame tronquée et de quelques pièces osseuses ouvragées. II faut aussi mentionner la présence, sur un assez bon nombre d'ossements de grands mammifères, de traces (incisions, entailles) de désarticulation et de décarnisation, signes indubitables que des groupes humains sont en partie responsables de l'accumulation de la composante faunique tardiglaciaire du gisement (CINQ-MARS, 1979; MORLAN et CINQMARS, 1982).

\section{DESCRIPTION DE LA VÉGÉTATION ACTUELLE}

Comme nous l'avons déjà signalé, le milieu qui nous intéresse est celui de la forêt boréale située en zone montagneuse. Autour des crêtes et des affleurements calcaires, les versants sud, bien drainés, sont peuplés d'épinettes blanches (Picea glauca); le couvert arbustif et herbacé, riche en éléments floristiques, est dominé

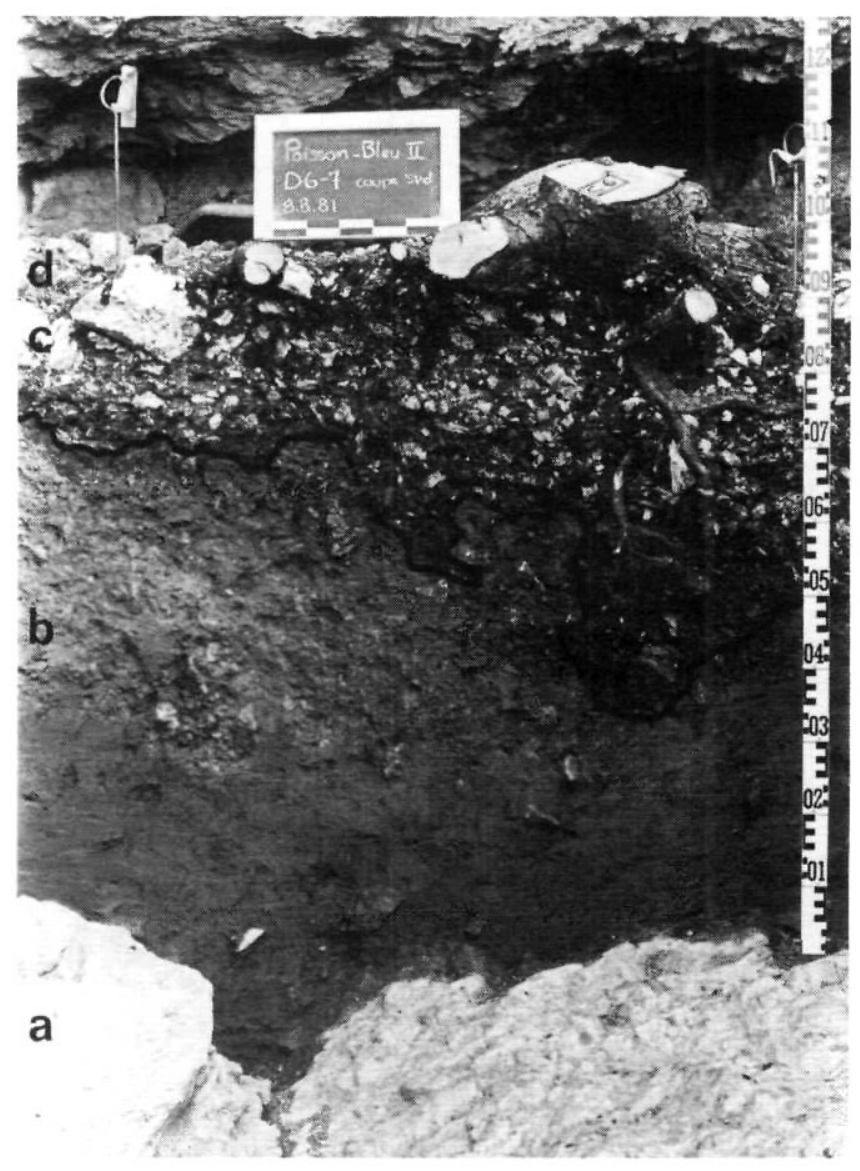

FIGURE 4. Coupe stratigraphique transversale d'une partie du gisement de la grotte II, illustrant la roche en place (a), la couche de lœss à cailloutis (b) ainsi que l'humus à cailloutis (c) et le sol récent (d).

Transverse stratigraphic profile of part of the Cave II deposit, showing the underlying bedrock (a), the stoney loess (b), the stoney humus (c), and the modern soil zone (d).

par Dryas octopetala, Salix reticulata, Carex scirpoidea, Cassiope tetragona, Arctostaphylos rubra et Tomenthypnum nitens. Quant aux versants, cuvettes et dénivellations exposés au nord, ils sont aussi couverts par une forêt d'épinette, mais dont la composition floristique est très différente. Ainsi, on n'y trouve que l'épinette noire (Picea mariana) accompagnée dans les strates arbustive et herbacée, sur les sites les plus humides, par Eriophorum vaginatum et Carex lugens et sur les sols plus secs, par Ledum groenlandicum, Vaccinium uliginosum et Rhododendron lapponicum.

La plaine alluviale de la rivière du Poisson-Bleu présente un couvert d'épinettes blanches dont certaines peuvent atteindre jusqu'à $17 \mathrm{~m}$ de hauteur et 20 à $30 \mathrm{~cm}$ de diamètre; associées à ces dernières se retrouvent aussi d'importantes concentrations d'aulnes (Alnus incana) ainsi que Shepherdia canadensis, Rosa beauverdiana, Linnaea borealis, Pyrola secunda et Viburnum edule. Le peuplier baumier (Populus balsamifera) de mê- 
me que diverses espèces de saules (Salix) sont aussi communs sur ces sols alluviaux.

Les crêtes au dessus de $750 \mathrm{~m}$ d'altitude sont dépouillées d'arbres et caractérisées par un paysage de toundra, dominé par Dryas octopetala, Hedysarum mackenzii, Salix reticulata, Arctostaphylos rubra, Saxifraga tricuspidata et Carex scirpoidea, associés à un riche assemblage de mousses et de lichens. Finalement, la région des grottes du Poisson-Bleu ne contient que de très rares trembles (Populus tremuloides), tandis que le bouleau de l'Alaska (Betula neoalaskana) et le mélèze (Larix laricina) en sont absents.

\section{ANALYSE POLLINIQUE}

Au cours de la deuxième campagne de fouille, en 1979, une série d'échantillons provenant de divers puits de sondage furent prélevés. Les échantillons qui font le sujet de cette étude ont été obtenus de la coupe est du puits G4 de la grotte II, où ils ont été recueillis à $2 \mathrm{~cm}$ d'intervalle, à partir de la surface jusqu'aux sédiments de la base recouvrant la roche en place gélifractée. Les sédiments prélevés entre 0 et $52 \mathrm{~cm}$ correspondent à l'humus à cailloutis décrit plus haut et se composent d'humus fibreux et de fragments de calcaire à coloration brune (10 YR 4/3); ceux recueillis entre 52 et 102 cm correspondent au lœss, gris pâle (10 YR 6/1) à cailloutis.

Tous les échantillons ont été préparés selon la méthode de FAEGRI et IVERSEN (1975, p. 107) et ils ont de plus été soumis au traitement de concentration des grains de pollen, développé récemment par CWYNAR et al. (1979). Ceci nous a permis d'identifier, en tout, 48 taxons et, bien que les concentrations polliniques aient été relativement faibles et que la fréquence des grains non identifiables ait été plutôt élevée, nous avons pu obtenir un nombre suffisant d'échantillons polliniques pouvant servir à établir un diagramme des pourcentages du pollen des types nécessaires à l'interprétation de l'histoire de la végétation (fig. 5).

La biostratigraphie essentielle qui ressort de cette analyse confirme et complète les résultats obtenus antérieurement à partir de l'étude des sédiments de la grotte I (CINQ-MARS, 1979, fig. 12). De $102 \mathrm{~cm}$ à 80 $\mathrm{cm}$, le diagramme illustre un assemblage dominé par des types de grains de pollen non arboréens, particulièrement des graminées (Gramineae) et des laiches (Cyperaceae), riche en espèces herbacées. De $80 \mathrm{~cm}$ à 50 $\mathrm{cm}$, les grains de bouleau (Betula), et les laiches atteignent une représentation maximale. Enfin, de $50 \mathrm{~cm}$ à 0 $\mathrm{cm}$, on peut noter une représentation importante de spores de Sphagnum, une diminution des taxons herbacés, et une nette augmentation de la courbe de l'épinet- te et de l'aulne. L'assemblage inférieur $(102 \mathrm{~cm}$ à 80 $\mathrm{cm}$ ) se compose de taxons dont la répartition actuelle est principalement arctique (Phlox, Pedicularis, Polygonum alaskanum, Aconitum, Hedysarum et Oxytropis). Ces taxons sont tous typiques de la toundra moderne. Les différents niveaux ont aussi fourni de forts pourcentages de spores pré-quaternaires qui, nous le supposons, proviennent de la désintégration des rochers environnants ou encore des sédiments éoliens eux-mêmes. Ces pourcentages élevés de lycopodes (Lycopodium) et de fougères (Filicales) sont en effet difficiles à expliquer autrement que par l'action de facteurs éoliens et sédimentaires. On trouve bien, aujourd'hui, quelques fougères dans les grottes (par exemple, Cystopteris fragilis et Woodsia ilvensis), mais elles ne peuvent pas être utilisées comme éléments comparatifs puisque les spores provenant des échantillons ne peuvent être identifiées au niveau du genre à cause de l'absence de périne.

Deux datations au ${ }^{14} \mathrm{C}$ (CINQ-MARS, 1979; MORLAN et CINQ-MARS, 1982), obtenues à partir du collagène d'os de grands mammifères (mammouth et cheval) recueillis dans la couche de lœss à cailloutis indiquent que la plus grande partie du remplissage éolien sur les replats en face des abris a été mis en place entre $15500 \pm 130 \mathrm{BP}(\mathrm{GSC}-3053)$ et $12900 \pm \mathrm{BP}$ (GSC 2881).

Notre stratigraphie pollinique semble être en accord avec cette chronologie. En effet, la zone inférieure du gisement du Poisson-Bleu peut être mise en corrélation avec la zone herbacée de Doll Creek (diagramme Lateral Pond, fig. 6), datée, elle-même, à $15200 \pm 230$ ans BP.

Cette ressemblance entre le diagramme pollinique du Poisson-Bleu et celui de Lateral Pond, à Doll Creek (fig. 6) est d'autant plus significative que les deux sites présentent de nombreuses et nettes analogies au niveau des facteurs écologiques. En outre, le diagramme de la concentration pollinique du Poisson-Bleu (nous n'illustrons ici que la concentration globale) et celui de Lateral Pond (RITCHIE, 1981, fig. 8) présentent de grandes ressemblances, indiquant par là qu'ils enregistrent les mêmes changements de végétation. Finalement, les assemblages polliniques enregistrés par CWYNAR (1982) à Hanging L., pour la période allant de 16000 à 14000 ans BP, ressemblent nettement, qualitativement et quantitativement, à la zone herbacée du Poisson-Bleu.

La zone moyenne (de $80 \mathrm{~cm}$ à $50 \mathrm{~cm}$ ) est comparable à la zone pollinique herbacées-bouleau de Hanging L. et de Lateral Pond, sauf qu'ici les donnees du Poisson-Bleu relatives au bouleau ne dépassent pas $40 \%$, tandis qu'aux autres sites, elles atteignent $80 \%$. II est probable que cette différence résulte de la préférence qu'a ce taxon pour les sols acides qui, faut-il le mentionner, sont absents dans le voisinage des grottes du 
Grotte du Poisson-Bleu II, Yukon septentrional

Pourcentages polliniques

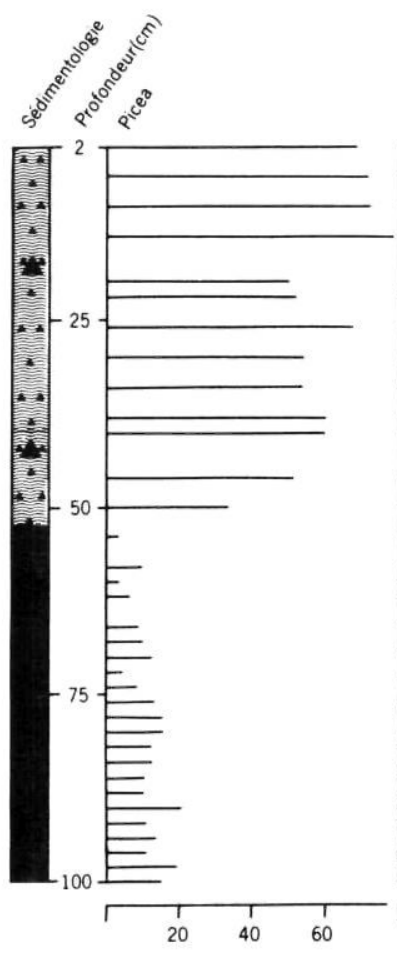

Humus/cailloux
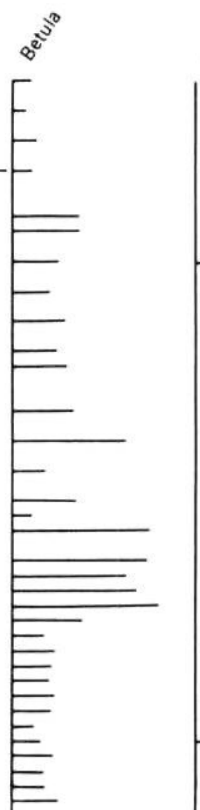

$\Gamma_{5}$
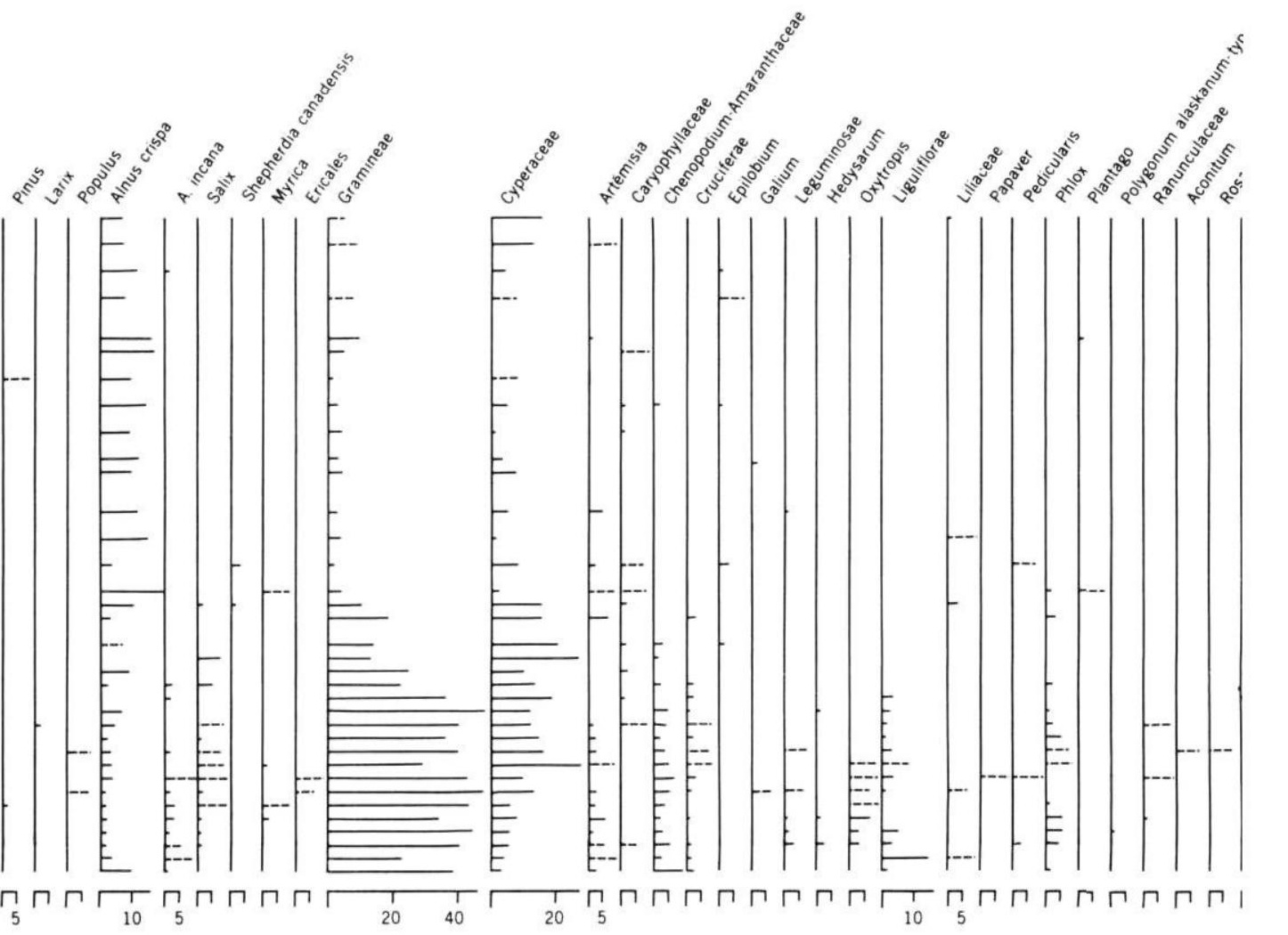

FIGURE 5. Diagramme des pourcentages polliniques et courbe de la concentration pollinique de la grotte II.

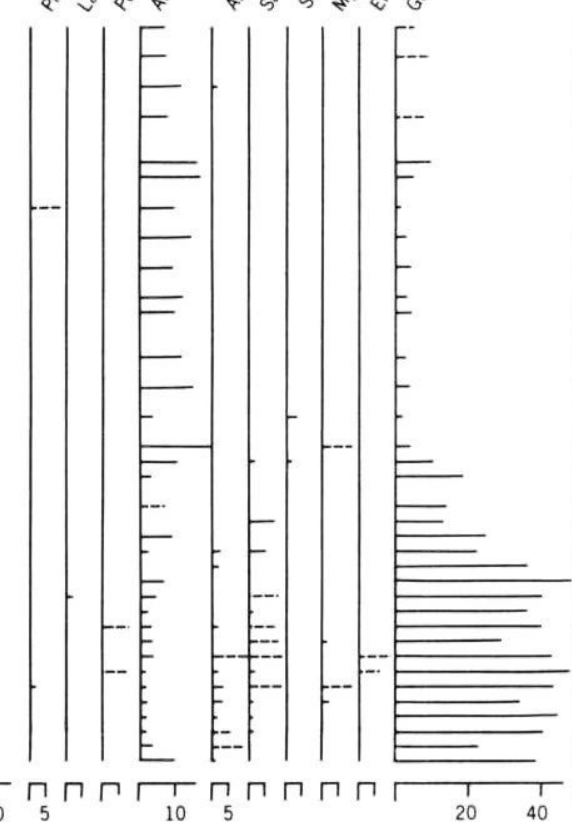

A Roches

A percentage pollen diagram of the Cave II sediments, including a total concentration curve.

Poisson-Bleu. D'ailleurs, la végétation de cette région ne contient que très peu de bouleaux arbustifs et, comme nous.l'avons déjà dit, le bouleau arborescent n'y croît pas.

Il est intéressant de constater que la partie supérieure du diagramme du Poisson-Bleu (de $50 \mathrm{~cm}$ à $0 \mathrm{~cm}$, fig. 5) illustre les mêmes traits polliniques que ceux qu'on retrouve dans les niveaux équivalents des diagrammes de Hanging L. et de Lateral Pond. Même si ces trois sites sont situés approximativement à la même latitude, au sein de la zone boréale, on aurait pu s'attendre à de plus grandes différences entre un diagramme provenant d'une grotte et des diagrammes de sédiments lacustres ou de tourbières. II €st bon de noter, cependant, que la séquence pollinique supérieure du PoissonBleu est nettement moins différenciée que celle qu'on peut observer dans les diagrammes des deux autres sites. Ainsi, l'époque de l'apparition de l'aulne (correspondant habituellement au dernier jalon stratigraphique de l'Holocène, en Béringie orientale) n'y est que faiblement enregistrée et, en fait, mal définie puisqu'elle semble coïncider avec la venue de l'épinette (Picea) et même se situer un peu plus tôt que cette dernière. Cet enregistrement, en apparence confus, ne devrait pas surprendre étant donné que les sédiments de la section supérieure du Poisson-Bleu se composent d'humus, de cailloux et de racines intrusives et qu'on peut y observer quelques traces de cryoturbation ainsi que des signes discontinus de discordance se situant au niveau de la transition lœss-humus.

Bien que peu commune, l'allure de la courbe de Sphagnum correspond probablement à un niveau normal de cette spore dans l'atmosphère de la région. En effet, des concentrations semblables à celles suggérées par cette courbe ont été trouvées dans des échantillons polliniques modernes obtenus au moyen d'un piège de type "Tauber" installé dans une des grottes. 

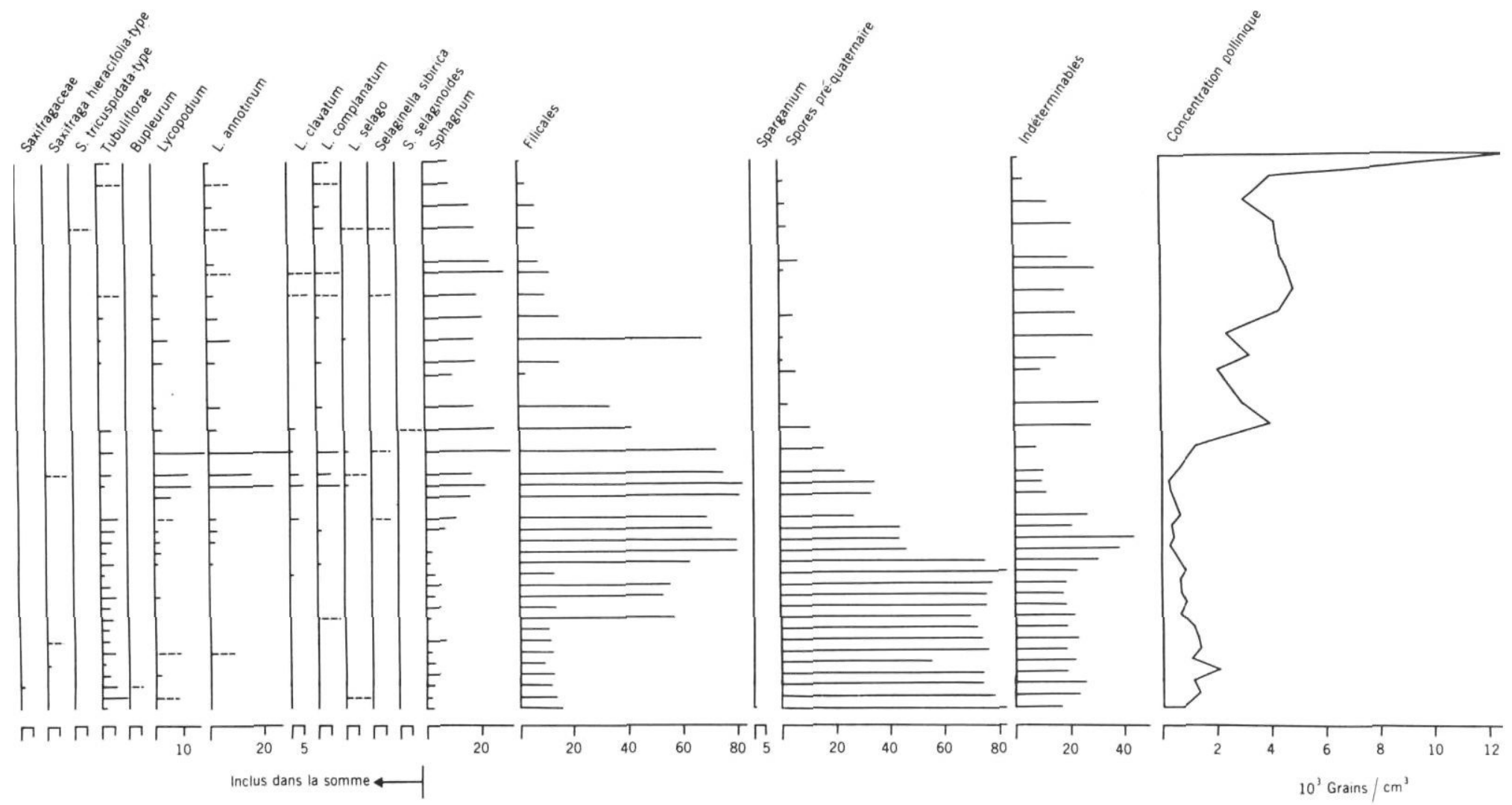

\section{RECONSTITUTION DE L'ENVIRONNEMENT}

Cette tentative de reconstitution de l'environnement de la Béringie orientale, entre environ 16000 ans BP et l'Holocène moyen est basée sur une série de données paléobotaniques pertinentes provenant des quatre sites mentionnés précédemment. Les données sont présentées de façon schématique au tableau I.

Le diagramme pollinique du Poisson-Bleu indique qu'une toundra herbacée a dominé pendant les deux ou trois premiers millénaires de la séquence sédimentaire, entre 16000 et 13000 ans BP. La concentration pollinique et la composition des espèces indiquent que les plateaux et les pentes élevées étaient couvertes d'une toundra éparse, semblable aux groupements végétaux qui existent aujourd'hui sur les crêtes exposées. D'autre part, il est probable que les vallées protégées par la neige en hiver et suffisamment humides en été, aient abrité des marais à laiches, graminées et saules.
Les données obtenues de Hanging L. et Doll Creek nous permettent de déduire que la période située entre 1600 et 13000 ans BP fut caractérisée par de faibles augmentations de l'influx pollinique provenant de ces groupements de toundra. A cette époque, les basses terres situées au nord de la crête du Poisson-Bleu étaient encore occupées par d'immenses lacs glaciaires, dits lacs de Bluefish et d'Old Crow (HUGHES, 1972), et il est probable que leur pourtour immédiat ait porté, à cause aussi de l'altitude de la nappe phréatique, des marécages à laiches et graminées ainsi que des carrs à saules.

Deux événements sont enregistrés, il y a environ 12800 ans BP. Tout d'abord, à Doll Creek, on trouve des témoignages polliniques reflétant probablement un épisode de refroidissement climatique. II semble que ce phénomène ait été d'importance régionale puisque, selon HAMILTON (1979), la dernière avancée des 


\section{Lateral Pond, Yukon septentrional}

Pourcentages polliniques

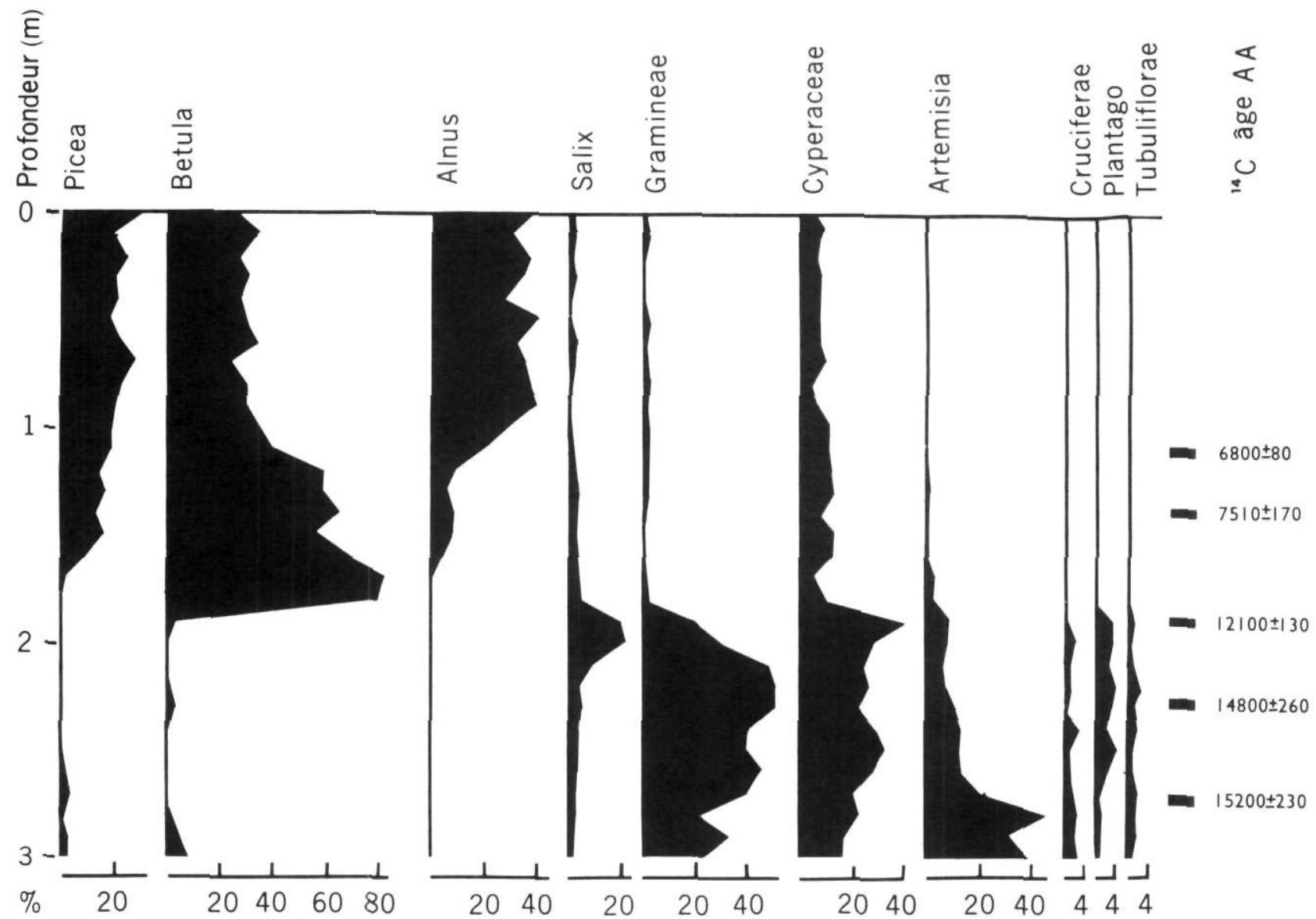

FIGURE 6. Diagramme pollinique simplifié des fréquences relatives du site de Lateral Pond (Doll Creek).

glaciers Itkillik II de la zone orientale des monts Brooks, en Alaska, se serait produite entre 12800 et 12500 BP. Enfin, au site Polybog, dans le bassin lacustre d'Old Crow, OVENDEN (1981) a décrit une succession de végétation, du type aquatique au type marécageux, qui s'est manifestée par suite de la vidange finale du lac glaciaire Old Crow, c'est-à-dire, vers 12500 BP.

À la suite de ces événements, il semble que des changements de végétation soient apparus très rapidement à tous les sites. Ceci est apparent dans les spectres polliniques par l'augmentation brusque du pollen de bouleau et ce, tant au niveau du pourcentage qu'à celui de l'influx. À ce sujet, il est bon de noter qu'en réalité, la très forte représentation (quadruple) du pollen de bouleau en a fait exagérer le rôle dans certaines reconstitutions végétales (HOPKINS, 1972). Les analyses détaillées de CWYNAR (1982), corroborées par celles de

A simplified relative pollen diagram from the Lateral Pond site (Doll Creek).

RITCHIE (1982) à Doll Creek, démontrent que l'accroissement des bruyères et la tourbification ont servi à transformer les toundras éparses de l'époque en une toundra plus dense, plus arbustive, semblable au type qui prédomine aujourd'hui sur de grandes surfaces. Le nombre de bouleaux sous-arbrisseaux s'est accru, mais ils n'ont jamais compté pour plus de $20 \%$ de la végétation, associés à Vaccinium, Arctostaphylos, Ledum, Empetrum, Eriophorum et Carex. A cette époque, tout comme aujourd'hui, ce type de bouleau semble avoir été très rare ou même absent des paysages calcaires comme celui du Poisson-Bleu. Les marais à laiches et à graminées ont été transformés en tourbières dominées par Sphagnum et les bruyères (OVENDEN, 1981). Finalement, le dernier événement correspond au développement et à l'établissement, au cours de l'Holocène ancien, des types modernes de forêt boréale, types qui n'ont subi que peu de changements jusqu'à nos jours. 
TABLEAU

Résumé de l'évolution de la couverture végétale

\begin{tabular}{|c|c|c|c|c|}
\hline$\underset{\mathrm{BP}}{\hat{\text { Age }}}{ }^{14} \mathrm{C}$ & Station Poisson-BLeu & $\begin{array}{l}\text { Station Doll Creek } \\
\text { (Lateral Pond) }\end{array}$ & Station Hanging L. & Station Polybog \\
\hline $\begin{array}{r}25000 \mathrm{a} \\
16000\end{array}$ & Absence de données. & Absence de données. & $\begin{array}{l}\text { Toundraherbacée,éparse } \\
\text { sur les hautes terres; } \\
\text { couverture de graminées } \\
\text { laiches et saules sur les } \\
\text { basses terres. }\end{array}$ & Lac glaciaire Old Crow. \\
\hline $\begin{array}{r}16000 \mathrm{à} \\
12800\end{array}$ & $\begin{array}{l}\text { Toundra herbacée sur les } \\
\text { hautes terres; graminées, } \\
\text { laiches et saules sur les } \\
\text { basses terres; croissance } \\
\text { des pourcentages de } \\
\text { bouleau; faune vertébrée } \\
\text { du Poisson-Bleu. }\end{array}$ & $\begin{array}{l}\text { Toundra herbacée sur les } \\
\text { hautes terres; marais à } \\
\text { graminées, laiches et } \\
\text { saules sur les basses ter- } \\
\text { res; augmentation d'in- } \\
\text { flux polliniques indiquant } \\
\text { un réchauffement gra- } \\
\text { duel du climat. }\end{array}$ & $\begin{array}{l}\text { Pourcentages croissants } \\
\text { de bouleaux arbustifs et } \\
\text { augmentation de la cou- } \\
\text { verture végétale herbeu- } \\
\text { se. }\end{array}$ & $\begin{array}{l}\text { Période de vidange } \\
\text { du lac glaciaire Old } \\
\text { Crow. }\end{array}$ \\
\hline $\begin{array}{r}12800 \text { à } \\
12000\end{array}$ & $\begin{array}{l}\text { Ibid.; début probable de } \\
\text { l'appauvrissement fauni- } \\
\text { que. }\end{array}$ & $\begin{array}{l}\text { Bref refroidissement indi- } \\
\text { qué par des diminutions } \\
\text { d'influx des herbes, des } \\
\text { saules et des graminées. }\end{array}$ & Ibid. & $\begin{array}{l}\text { Colonisation des sédi- } \\
\text { ments lacustres, glaciai- } \\
\text { res par une séquence de } \\
\text { végétation aquatique et } \\
\text { marécageuse. }\end{array}$ \\
\hline $\begin{array}{l}11500 \text { à } \\
\text { l'Holocène } \\
\text { moyen }\end{array}$ & $\begin{array}{l}\text { Époque de la pessière; } \\
\text { extinction ou disparition } \\
\text { de la faune tardiglaciaire. }\end{array}$ & $\begin{array}{l}\text { Développement rapide de } \\
\text { la toundra à bruyères et } \\
\text { à bouleaux arbustifs; } \\
\text { apparition de la pes- } \\
\text { sière, conséquence d'un } \\
\text { climat plus chaud et plus } \\
\text { humide. }\end{array}$ & $\begin{array}{l}\text { Apparition de la bruyère } \\
\text { humide; augmentation } \\
\text { élévée d'influx indiquant } \\
\text { un réchauffement rapide } \\
\text { du climat. }\end{array}$ & $\begin{array}{l}\text { Succession graduelle de } \\
\text { végétation lacustre, à } \\
\text { marécages et à tourbières } \\
\text { à dominance de sphai- } \\
\text { gnes et de bruyères. }\end{array}$ \\
\hline
\end{tabular}

\section{CONCLUSION}

Afin de résumer l'histoire de la végétation de la région des grottes du Poisson-Bleu, il est possible, tout d'abord, de supposer - en extrapolant à partir des résultats de l'analyse de Hanging $L$. - que pendant le pléniglaciaire (autour de $18000 \mathrm{BP}$ ), un climat froid et sec a permis l'existence, sur les plateaux et les hautes terres, d'une végétation éparse de toundra herbacée avec, dans les basses terres, des groupements très localisés de saules et des marécages à laiches et à graminées. À partir de ces données, nous croyons qu'il est toujours possible de supposer (CWYNAR et RITCHIE, 1980) que le pléniglaciaire du Yukon septentrional était caractérisé par un appauvrissement régional de la couverture végétale et même, possiblement, par une absence locale bien que temporaire de certaines composantes de la faune vertébrée.

Par la suite, entre 16000 et 12000 BP, le climat glaciaire se réchauffant lentement, une toundra herbacée un peu plus complexe et plus productive s'est développée, en région de plateaux et de hautes terres, tandis que des marais et des peuplements denses de saules se sont établis dans les vallées et les plaines alluviales. Les données paléontologiques et culturelles obtenues du gisement du Poisson-Bleu indiquent clairement qu'un tel écosystème, à structure en mosaïque, a pu soutenir, tout au moins pendant quelques millénaires, la faune tardiglaciaire mentionnée précédemment et les groupes humains qui semblent avoir exploité les principaux éléments de cette faune (CINQ-MARS, 1979; MORLAN et CINQ-MARS, 1982).

Vers $11500 \mathrm{BP}$, la végétation s'est rapidement transformée: de toundra herbacée en toundra arbustive sur les plateaux et les pentes, et de marais et saulaies en tourbières dans les vallées et les basses terres. Ce changement est interprété comme un réponse du couvert végétal au passage brusque d'un climat glaciaire froid et sec à un climat interglaciaire plus chaud et moins sec. L'extinction ou la disparition locale ou régionale des principaux éléments de la faune tardiglaciaire a coïncidé avec cet événement important. Mais il n'existe pas, pour le moment, de témoignages directs qui puissent nous permettre de vérifier et de préciser les nombreuses spéculations relatives aux causes de cet appauvrissement faunique. 


\section{REMERCIEMENTS}

Ce rapport résulte de travaux de recherche effectués dans le cadre du Northern Yukon Research Programme de l'université de Toronto, subventionné par le Conseil de recherche en sciences humaines du Canada, par l'université de Toronto, par la Donner Foundation et par le ministère des Affaires indiennes et du Nord, et grâce à la subvention A6320, fournie par le Conseil de recherches en sciences naturelles et en génie du Canada à J.C. Ritchie. Des données additionnelles ont aussi été obtenues grâce à la participation de la Commission archéologique du Canada, Musée national de l'Homme, Musées nationaux du Canada. Nous remercions tout particulièrement Mlle K. Hadden qui a effectué les analyses polliniques.

\section{RÉFÉRENCES}

CINQ-MARS, J. (1979): Bluefish Cave I: a Late Pleistocene eastern Beringian cave deposit in the northern Yukon, Journ. can. Archéol. vol. 3, p. 1-32.

CWYNAR, L.C. (1982): A Late-Quaternary vegetation history from Hanging Lake, Northern Yukon, Ecol. Monogr., sous presse.

CWYNAR, L.C. BURDEN, E. et MCANDREWS, J.H. (1979) : An inexpensive sieving method for concentrating pollen and spores from fine-grained sediments, Can. J. Earth Sci., vol. 16 , p. $1115-1120$.

CWYNAR, L.C. et RITCHIE, J.C. (1980): Arctic Steppe-Tundra: a Yukon Perspective, Science, $n^{\circ} 208$, p. 1375-1377.

FAEGRI, K. et IVERSEN, J. (1975): Textbook of Pollen Analysis, $3^{e}$ éd., Munksgaard, Copenhague, 295 p.
HAMILTON, T.D. (1979): Radiocarbon dates and Quaternary stratigraphic sections, Philip Smith Mountains quadrangle, Alaska, U.S. Geol. Surv., Open File Report 79-866, 44 p.

HARINGTON, C.R. (1978): Quaternary vertebrate faunas of Canada and Alaska and their suggested chronological sequence, Syllogeus, $n^{\circ} 15$, Musées nationaux du Canada, Ottawa, $105 \mathrm{p}$.

HOPKINS, D. M. (1972) : The palaeogeography and climatic history of Beringia during Cenozoic times, Inter-Nord, vol. 12, p. 121-150.

HUGHES, O. (1972): Surficial geology of northern Yukon Territory and northwestern District of Mackenzie, Northwest Territories, Paper 69-36, Comm. géol. Can., Ottawa, 11 p.

LUMLEY, H. de, MISKOVSKY, J.-C., RENAULT-MISKOVSKY, J. et GERBER, J.-P. (1973): Le Würmien ancien dans le Midi méditerranéen d'après les dépôts de grottes, Le Quaternaire, Travaux français récents, L'INQUA IX 19, 79-89. 239 p.

MORLAN, R.E. (1980): Taphonomy and archaeology in the Upper Pleistocene of the Northern Yukon Territory: a glimpse of the peopling of the New World, Coll. Mercure, Dossier $\mathrm{n}^{\circ}$ 94, Comm. archéol. Can., $398 \mathrm{p}$.

MORLAN, R.E. et CINQ-MARS, J. (1982) : Ancient Beringians: human occupations in the Late Pleistocene of Alaska and the Yukon Territory, in Palaeoecology of the Arctic Steppe-Mammoth Biome, publié sous la dir. de D.M. Hopkins. Academic Press, New York, sous presse.

OVENDEN, L. (1981): vegetation history of a polygonal peatland, Old Crow Flats, Northern Yukon, thèse M.Sc., Dept. of Botany, Univ. of Toronto, $83 \mathrm{p}$.

RENAULT-MISKOVSKY, J. (1978): La grotte du Vallonnet (Roquebrune Cap-Martin-Alpes-Maritimes); analyse pollinique du remplissage, Géol. méditerranéenne, vol. 2.

RITCHIE, J.C. (1982): The modern and Late-Quaternary vegetation of the Doll Creek area, N. Yukon, Canada, New Phytologist, vol. $90 n^{\circ} 3$, sous presse. 